\title{
Celiac Crisis Presenting with Refractory Hypokalemia and Bleeding Diathesis
}

\author{
Mehta S, Jain J, Mulye S
}

Department of Pediatrics

Sri Aurobindo Medical College \& PG Institute

Indore, Madhya Pradesh, India

\begin{abstract}
Celiac disease is an immune mediated disorder elicited by the ingestion of gluten in genetically susceptible person and associated with some HLA subtypes. It is characterized by chronic inflammation of small intestine associated with villous atrophy. Symptoms usually appear when weaning from breast milk is done and gluten is introduced in diet. Failures to thrive, chronic diarrhea, vomiting abdominal distension are usual symptoms. Celiac crisis is rare but well known complication of celiac disease presenting with various systemic and metabolic manifestations like hypokalemia, hyponatremia, shock and bleeding diathesis. Celiac crisis usually has some underlying precipitating factor and is more common in patient with noncompliance with gluten free diet. We report a case of 14 year and three months old female child, a known case of celiac disease having the poor compliance with gluten free diet, presenting in celiac crisis with shock, refractory hypokalemia and bleeding diathesis.
\end{abstract}

Mehta S, Jain J, Mulye S. Celiac Crisis Presenting with Refractory Hypokalemia and Bleeding Diathesis. Kathmandu Univ Med J 2014;48(4):296-7.

\section{KEY WORDS}

Bleeding diathesis, celiac crisis, refractory hypokalemia

\section{INTRODUCTION}

Celiac disease or gluten enteropathy is an autoimmune mediated disorder elicited by the ingestion of gluten in genetically susceptible person and is characterized by chronic inflammation of small intestine associated with villous atrophy. ${ }^{1}$ Celiac crisis is a fatal complication of celiac disease. It may present as acute exacerbation in known celiacs or may be the first presentation of the disease. There are constellation of symptoms including profound diarrhea with dehydration, weight loss, shock with metabolic acidosis, hypocalcemia, hyponatremia, hypoproteinemia leading to severe metabolic consequences.

\section{CASE-REPORT}

A fourteen year and three months old female child, $2^{\text {nd }}$ live issue of non consanguineous marriage of healthy parents and born at term following uncomplicated pregnancy presented with frequent episodes of diarrhea after six months of age, when she was weaned from breast milk. She was diagnosed at age of $3^{1 / 2}$ year of age as a case of celiac disease. Diagnosis was made by serology-anti tissue transglutaminase antibody and confirmed by GI endoscopy biopsy showing marsh stage 3 changes. She was started on gluten free diet. However, she had poor compliance and had frequent admissions for diarrhea with dehydration including one with encephalopathy. Last admission was one month back. Anti tissue transglutaminase antibody titers were high last time and GI biopsy also suggested of celiac disease. This time she was admitted with acute onset of diarrhea and vomiting since two days. She had proportionate short stature ht $127 \mathrm{~cm}<-3$ SD of Z score percentile\}, weight $24 \mathrm{KG}$ [<-3 SD of Z score rd percentile], wt for ht <-2 2 SD with BMI of $14.6 \mathrm{~kg} / \mathrm{m}^{2}$ [<-2 SD]. She had dehydration with hypotension and shock. She had pallor, grade 1 clubbing and there were multiple erythematous scaly and eczematous lesions with areas of denudation over both flexor and extensor surfaces. On investigations Complete blood count revealed anemia with hemoglobin $8.2 \mathrm{~g} / \mathrm{dl}$, total leukocyte count 9400 (polymorphs $79 \%$, leucocyte $18 \%$, eosinophils $2 \%$, monocyte $1 \%$ ), thrombocytopenia with platelet count 16800 , peripheral smear showing dimorphic anemia. Serum sodium -134 $\mathrm{meq} / \mathrm{l}$, hypokalemia with potassium $-2.1 \mathrm{meq} / \mathrm{l}$, chloride -112 meq/l, random blood sugar $-106 \mathrm{mg} / \mathrm{dl}$, Magnesium $-2 \mathrm{meq} / \mathrm{l}$, serum phosphorus $-4.2 \mathrm{mg} / \mathrm{dl}$, arterial blood gas showing metabolic acidosis ( $\mathrm{PH}-7.12$, po2 -66, pco2 -36, 


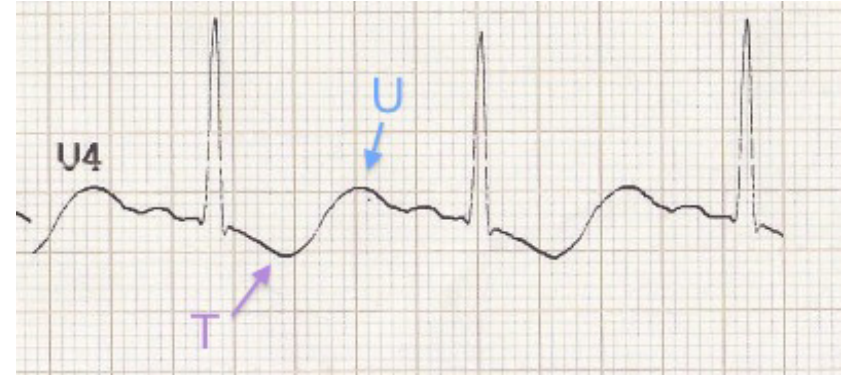

Figure 1. ECG picture showing ST segment depression, appearance of $U$ wave (features suggestive of hypokalemia).

HCO3 -4.2 meq/l), anti tissue transglutaminase antibody titres were significantly high, initial chest $X$ ray was normal. Patient was admitted in pediatric intensive care unit, managed for shock with IV fluid boluses and inotropic support, oxygen inhalation required soda bicarbonate correction for metabolic acidosis, potassium supplementation up to $80 \mathrm{meq} / \mathrm{l}$ in IV fluids for persistent hypokalemia and given 3 platelets concentrates and was started on IV steroids. Repeat Complete blood count after 24 hours -Hemoglobin $-6 \mathrm{~g} / \mathrm{dl}$, total leucocyte count $6800 \mathrm{~mm}^{3}$, thrombocytopenia with platelets 7800 , repeat serum sodium $132 / 138$ and potassium -2.2/1.8. ECG (figure-1) was suggestive of evidence of hypokalemia in form of flat T wave, depressed ST segment, appearence of $U$ wave and blood culture revealed no growth. She was again given one fresh whole blood, 3 more platelet infusions. Her condition was not responding to treatment. Hypokalemia and shock became refractory. She had evidence of multiple organ dysfunction syndromes, involving cardiovascular, respiratory, hematological and CNS with GCS kept on deteriorating. She required ventilator support; developed manifestations of thrombocytopenia in form of bleeding from GI tract and had pulmonary hemorrhage. She succumbed to the illness.

\section{DISCUSSION}

Celiac crisis is rare but well known complication of celiac disease presenting with various systemic and metabolic manifestations like refactory hypokalemia, hyponatremia and shock. ${ }^{2}$ There is acute onset and rapid progression of GI symptoms with signs and symptoms of dehydration and

\section{REFERENCES}

1. Farrell RJ, Kelly CP. Celic sprue. N Eng/ J Med 2002;346:180-8

2. Baranwal AK, Singhi SC, Thapa BR, Kakkar Nel. Celiac crisis. Ind Pediatr $2003 ; 70: 433-5$

3. Steens RF, Csizmadia CG, Georage EK, Nianaber MK, Hira Sing RA, Mearin MLI. A national prospective on childhood celiac disease in Netherlands 1993-2000: An increasing recognition and a changing clinical picture. J Pediatr 2005;147:239-43

4. Puri AS, Garg S, Monga R, Tyagi P, Saraswat MK. Spectrum of Atypical celiac disease in North Indian Children. Indian Pediatr 2004;41:822-7

5. Hill ID, Dirks MH, Liptak GS. Guidelines for diagnosis and treatment of celiac disease in children: Recommendations of North American society for Pediatric Gasteroenterology, Hepatology and Nutrition. J Pediatr Gastroenteral Nutr 2005; 40: 1-19 malnutrition. It may be associated with other autoimmune manifestations like thrombocytopenia. Celiac disease is immune mediated disorder having a genetic basis with sensitivity to gluten in diet. It is a chronic inflammatory disorder associated with certain HLA subtypes DQA1 05 and DQB1 02. Genome wide association studies have shown risk variants in genes controlling immune response, some being shared with type 1 diabetes and other autoimmune disorders. The most evident expression of autoimmunity is presence of serum antibodies to transglutaminase. Several extra intestinal clinical manifestations of celiac disease [e.g. liver, heart, nervous system] are possibly related to antibodies. Symptoms usually appear, when weaning from breast milk is done and gluten is introduced in diet. Failure to thrive, chronic diarrhea, vomiting abdominal distension, muscle wasting and anorexia are present in most of cases. ${ }^{3}$ As the age of presentation shift to later in childhood, extra intestinal manifestations become increasingly recognized, these includes iron deficiency anemia, short stature, arthritis and arthralgia, peripheral neuropathies, cardiomyopathy, endocrinopathy, encephalopathy, psychosis, sleep disturbances and other autoimmune manifestations. ${ }^{4,5}$ Diagnosis is made by serology and confirmed by histology changes in biopsy specimens from endoscopy. The histological changes are graded according to Marsh criteria, grade $3 / 4$ changes are characteristics of celiac disease and grade 2 changes need positive serology. ${ }^{6}$ Treatment is lifelong gluten free diet. After initiation of gluten free diet, there is a prompt response with rapid gain in weight and height within few months. There are few such case report of celiac crisis presenting as severe metabolic disturbances and shock requiring as intensive care management and systemic steroids. ${ }^{7,8}$ The constellation of clinical features reported and metabolic disturbances should prompt the clinician to investigate for the celiac crisis especially in poor compliant patient. Celiac crisis presenting as shock requires urgent intensive care management and correction of various metabolic disturbances and various bleeding diathesis. There is a role of systemic steroids .Delay in recognition of celiac state and poor compliance in already known case of celiac disease leads to very high mortality. The exact incidence of celiac crisis and mortality is not known. A study done by Barbar et al, found $25 \%$ incidence of celiac crisis. ${ }^{9}$ But it has been considered rare in many studies. ${ }^{2,10,11}$ In modern era mortality has been reduced due early recognition of symptoms of celiac crisis and effective implementation of gluten free diet.

6. Fasano A, Catassi C. Current approaches to diagnosis and treatment of celiac disease: an evolving spectrum. J Gastroenteral 2001;120:63651

7. Lloyd-still Jd, Grand RJ ,Khaw KT, Shwachman H. The use of corticosteroids in celiac criss. J Pediatr 1972;81:1074-1081

8. Walia A, Thapa BR. Celiac crisis. Indian Pediatr 2005;42:116-9

9. Barbar MI, Ahmad I, Rao MS, Iqbal R, Asghar S, Saleem S. Celiac disease and celiac crisis in children. J Coll Physicians Surg Pak 2011; 21 (8):487-90

10. Peter G, Christopher C. Celiac disease. N Engl J Med 2007; 357: 1731-43

11. Jamma S, Rubio-Tapia A, Kelly CP, Murray J, Sheth S, Schuppan D etal. Celiac crisis is a rare but serious complication of celiac disease in adults. Clin Gasteroenteral Hepatol 2010;8:587-590 